\title{
NÍVEIS CRÍTICOS DE FÓSFORO NO SOLO E NA PLANTA PARA GRAMÍNEAS FORRAGEIRAS TROPICAIS, EM DIFERENTES IDADES ${ }^{(\mathbf{1})}$
}

\author{
H. Q. SANTOS ${ }^{(2)}$, D. M. FONSECA(3), R. B. CANTARUTTI ${ }^{(4)}$, \\ V. H. ALVAREZ V. ${ }^{(4)} \&$ D. NASCIMENTO J ÚNIOR ${ }^{(3)}$
}

\begin{abstract}
RESUMO
Dois experimentos foram realizados simultaneamente, em casa de vegetação, para avaliar os efeitos da idade das plantas e de períodos de rebrotação sobre os valores dos níveis críticos de fósforo no solo e na planta para duas gramíneas forrageiras tropicais. Os tratamentos corresponderam aos arranjos fatoriais $2 \times 5 \times 5$, no estudo de crescimento inicial, e $2 \times 2 \times 5$, no estudo de rebrotação. Os fatoriais consistiram de duas espécies (Brachiaria decumbens cv. Basilisk e Panicum maximum cv. Mombaça); cinco idades (14, 28, 42, 56 e 70 dias após a emergência), durante o crescimento inicial e em dois períodos do estudo da rebrotação ( 28 e 42 dias) após um crescimento inicial de 42 dias, e cinco doses de fósforo $\left(0,64,128,320\right.$ e $\left.640 \mathrm{mg} \mathrm{dm}^{-3}\right)$. Os tratamentos, com três repetições, foram distribuídos em blocos ao acaso. Nos ensaios, utilizou-se amostra de Latossolo Vermelho-Amarelo. Para determi nação dos teores de fósforo disponível no solo, foi utilizado o extrator Mehlich-1. Os níveis críticos (NíCrí) de fósforo no solo para B. decumbens foram maiores do que para P. maximum. Os NíCrí no solo decresceram exponencialmente com a idade das plantas de 95,1 e 75,6 $\mathrm{mg} \mathrm{dm}^{-3}$, aos 14 dias, para 38,1 e $28,4 \mathrm{mg} \mathrm{dm}^{-3}$, aos 70 dias, com relação a B. decumbens e P. maximum, respectivamente. Na fase de rebrotação, os NíCrí no solo foram semelhantes nos dois períodos, com valores próximos aos estimados para as plantas aos 70 dias. Os NíCrí de fósforo na parte aérea das plantas decresceram exponencialmente de 0,57 e 0,60 dag kg-1, aos 14 dias, para 0,08 e 0,07 dag gg-1, $^{-1}$, aos 70 dias, com relação a $B$. decumbens e $P$. maxi mum, respectivamente. $B$. decumbens apresentou maiores valores de NíCrí de fósforo na parte aérea das plantas, exceto na idade de $\mathbf{1 4}$ dias. $\mathbf{N a}$ fase de rebrotação, os NíCrí na parte
\end{abstract}

(1) Parte da Tese de Mestrado do primeiro autor, apresentada ao Departamento de Zootecnia da Universidade Federal de Viçosa UFV. Recebido para publicação em abril de 2001 e aprovado em setembro de 2001.

(2) Doutorando em Solos e Nutrição de Plantas do Departamento de Solos, Universidade Federal de Viçosa - UFV. Av. P.H. Rolfs, s/n, CEP 36571-000 Viçosa (MG). Bolsista do CNPq. E-mail: hquadros@pobox.com

(3) Professor do Departamento de Zootecnia, UFV. Av. P.H. Rolfs s/n, CEP 36571-000 Viçosa (MG). Bolsistas do CNPq. E-mails: dfonseca@mail.ufv.br; domicio.nascimento.jr@ufv.br

(4) Professor do Departamento de Solos, UFV. Bolsistas do CNPq. E-mails: cantarutti@solos.ufv.br; vhav@mail.ufv.br 


\begin{abstract}
aérea foram maiores aos $\mathbf{2 8}$ dias, diminuindo, aos $\mathbf{4 2}$ dias, para valores similares aos estimados nas idades de 56 e $\mathbf{7 0}$ dias, no crescimento inicial. Assim, para avaliação criteriosa do estado nutricional da planta em termos de fósforo e recomendação de fertilização, recomenda-se consi derar a idade das plantas após o plantio da forrageira e a cada ciclo de crescimento.
\end{abstract}

\author{
Termos para indexação: Pani cum maximum, Brachiaria decumbens, Mombaça, \\ pastagem.
}

\title{
SUMMARY: SOIL AND PLANT PHOSPHORUS CRITICAL LEVELS FOR TROPICAL FORAGE GRASSES AT DIFFERENT AGES
}

\begin{abstract}
Two experiments werecarried out simultaneousl y in a greenhouseto evaluatetheeffects of plant ageand regrowth periods on soil and plant phosphorus critical levels for two tropical foragegrasses. Treatments corresponded to the $2 \times 5 \times 5$ factorial arrangement in theinitial growth study, and the $2 \times 2 \times 5$ factorial in the regrowth study. Thosefactorials consisted of two species (Brachiaria decumbens cv. Basilisk and Panicum maximum cv. Mombaça); fiveplant ages $(14,28,42,56$, and 70 days after emergence) in theinitial growth and during two periods of regrowth ( 28 and 42 days) after an initial growth of 42 days; and fivedoses of phosphorus $\left(0,64,128,320\right.$, and $\left.640 \mathrm{mg} \mathrm{dm}^{-3}\right)$. Treatments were distributed in a randomized block design with three replications. A Red-Yellow Latosol sample was used. Mehlich-1 extractant was used to determine soil phosphorus availability. Soil phosphorus critical levels weregreater for B. decumbens than for P. maximum. Soil phosphorus critical levels decreased exponentially with plant ages from 95.1 and $75.6 \mathrm{mg} \mathrm{dm}^{-3}$, at 14 days and to 38.1 and $28.4 \mathrm{mg} \mathrm{dm}^{-3}$ at 70 days, for B. decumbens and P. maximum, respectively. Soil phosphorus critical levels weresimilar in both regrowth periods with values close to those estimated for 70 day old plants. Phosphorus critical levels in plant shoot decreased exponentially with plant age from 0.57 and $0.60 \mathrm{dag} \mathrm{kg}^{-1}$, at 14 days, to 0.08 and $0.07 \mathrm{dag} \mathrm{kg}^{-1}$, at 70 days, for $B$. decumbens and $P$. maximum, respectively. B. decumbens had greater values of plant phosphorus critical levels except for 14-day-old plants. In the regrowth experiment, the phosphorus critical levels, in the shoots were greater for 28-day-old plants, but for 42-day-old plants the critical level s decreased to similar values of thoseesti mated at 56 and 70 days after emergence, in theinitial growth experiment. Therefore, it is necessary to evaluate plant age after its establishment and at each growing cycle to determine its nutritional status and to recommend phosphorus fertilization.
\end{abstract}

Index terms: Panicum maximum, Brachiaria decumbens, Mombaça, forages.

\section{NTRODUÇÃO}

No Brasil, a produção pecuária é predominantemente extensiva e sobre pastagens nativas ou cultivadas. Os índices zootécnicos da pecuária brasileira são baixos em razão, dentre outros fatores, da baixa qualidade e, principalmente, da pequena capacidade de suporte das pastagens. A baixa produtividade das forrageiras, ou a sua diminuição com o passar dos anos após o estabelecimento em solos tropicais, tem como causas principais: (a) a el evada acidez do solo ( $\mathrm{pH}<5,0)$, a toxidez de alumínio e manganês e a baixa disponibilidade de nutrientes, principal mente de fósforo e de nitrogênio (Rao et al., 1995); (b) o caráter extrativista com que se pratica a pecuária, ignorando-se a retirada de nutrientes do solo via produção animal (Zimmer e
Correa, 1993), e(c) oinadequado manejodas pastagens (Macedo, 1995).

O fósforo desempenha papel importante no crescimento do sistema radicular, bem como no perfilhamento das gramíneas, quesão fundamentais à maior produtividade das forrageiras. Embora o fósforo seja, de acordo com H ol ford (1997), o segundo nutriente essencial mais limitante à produção agrícola, depois do nitrogênio, nos solos tropicais, a "construção" da fertilidade do sol o em fósforo tornase particularmente importante, uma vez que esses solos apresentam baixa disponibilidade natural e alta capacidade de adsorção e de fixação desse nutriente. Somado a esses fatos, a absorção, assi milação etransl ocação de nitrogênio nas plantas podem ser restringidas pela deficiência de fósforo (Gniazdowska et al., 1999). 
Essas consi derações evidenciam que a adubação fosfatada é imprescindível à implantação e manutenção das forrageiras; no entanto, requer-se o estabel ecimento de critérios mais exatos para sua recomendação, visando a uma maior eficiência econômica da pecuária. A determinação dos níveis críticos de fósforo no solo e na planta é necessária para o estabel ecimento das doses de fósforo a serem aplicadas e a avaliação do estado nutricional das plantas. Esses níveis críticos variam com as espécies, condições edafoclimáticas (temperatura, luminosidade, disponibilidade hídrica, tipo de solo), disponibilidade de outros nutrientes, manejo da adubação (forma, tipo e época de aplicação de fertilizantes), forma de amostragem do sol o e idade da planta (Bates, 1971; Alvarez V., 1996).

Os níveis críticos de fósforo no solo e na planta diminuem com a idade das plantas (N ovais et al., 1982), sendo a variação mais acentuada nas plantas perenes, como é o caso da maioria das forrageiras. Assim, torna-se essencial estabelecer os níveis críticos de fósforo para cada um dos estádios de crescimento das plantas. Esse conhecimento tanto é imprescindível ao manejo da adubação, seja no plantio ou na manutenção, com vistas em suprir, dessa forma, a demanda das plantas ao longo do seu cicl o e favorecer a sustentabilidade da produção.

Com o presentetrabalho, objetivou-seestabelecer os níveis críticos de fósforo no sol oena planta conforme a idade das plantas, no caso deBrachiaria decumbens cv. Basilisk e Panicum maximum cv. Mombaça, cultivadas em um Latossolo Vermel ho-Amarelo.

\section{MATERIAL E MÉTODOS}

Amostra de um Latossolo Vermel ho-Amarelo textura argilosa (49\% de argila e 13\% de silte) foi coletada da camada de 0-20 cm, seca ao ar e passada em peneira com malha de $4 \mathrm{~mm}$ de abertura. A análise química indicou pH em água 5,2 e teores de $\mathrm{P}$ e $\mathrm{K}$ em Mehlich-1 de 2,5 e $35 \mathrm{mg} \mathrm{dm}^{-3}$, respectivamente. $\mathrm{O}$ val or do $\mathrm{P}$-remanescente ${ }^{(5)}$ foi de $15,5 \mathrm{mg} \mathrm{L}^{-1}$. A saturação por bases do solo era de $38 \%$. O trabalho constituiu-se de dois experimentos, realizados simultaneamente em casa de vegetação, em Viçosa (MG), no período de 6 de novembro de 1997 a 9 de abril de 1998.

\section{Experimento 1 - "crescimento inicial"}

Neste experimento, os tratamentos consistiram da combinação fatorial entre as duas espécies

\footnotetext{
${ }^{(5)}$ Fósforo remanescente (P-remanescente) é a concentração de fósforo da solução de equilíbrio, após agitar, durante uma hora, $5 \mathrm{~cm}^{3}$ deTFSA com $50 \mathrm{~mL}$ de solução de $\mathrm{CaCl}_{2} 10 \mathrm{mmol} \mathrm{L}^{-1}$, com $60 \mathrm{mg} \mathrm{L}^{-1}$ de fósforo (Alvarez V. et al., 2000).
}

forrageiras (Brachiaria decumbens Stapf. CV. Basilisk - BRA 001058 ePanicum maximumJ acq. cv. Mombaça - BRA 006645), cinco idades de crescimento (14, 28, 42, 56 e 70 dias após a emergência) e cinco doses de fósforo $\left(0,64,128,320\right.$ e $640 \mathrm{mg} \mathrm{dm}^{-3} \mathrm{de}$ fósforo), que foram determinadas com base no val or do P-remanescente do solo (Alvarez V. \& Fonseca, 1990). Os tratamentos foram distribuídos em blocos ao acaso, com três repetições.

O volume de $5,5 \mathrm{dm}^{3}$ de solo de cada uma das unidades experimentais recebeu a dose de cal cário dolomítico (PRNT =100,1\%) necessária para el evar a saturação por bases para 50\%, sendo acondicionado em vasos plásticos e permanecendo incubado por 30 dias com a umidade do solo mantida em $70 \%$ da capacidade de campo. Em seguida, as doses de fósforo foram aplicadas como superfosfato triplo (41\% de $\mathrm{P}_{2} \mathrm{O}_{5}$ solúvel em citrato neutro de amônio + água) triturado e com granulometria inferior a 0,25 mm, sendo homogeneizado com o solo das unidades experimentais. Seguiu-senovo período de 30 dias de incubação, mantendo-se a umidade do solo, como indicado anteri ormente.

Decorrido esse período, oito plântulas germi nadas e crescidas por cinco dias em leito de areia foram transplantadas para cada unidade experimental. Esse número de plantas foi mantido até o corte de aval iação 14 dias após a emergência, quando foi feito o desbaste, deixando-se quatro plantas por unidade experimental. Durante o período de crescimento das plantas, a umidade do solo foi mantida próxima à capacidade de campo, por meio de irrigações diárias.

As plantas receberam adubação com potássio, nitrogênio e enxofre por meio de soluções à base de $\mathrm{KNO}_{3},\left(\mathrm{NH}_{4}\right)_{2} \mathrm{SO}_{4}, \mathrm{KCl}$ e $\mathrm{CO}\left(\mathrm{NH}_{2}\right)_{2}$, aplicadas semanalmente. Desse modo, nos tratamentos correspondentes ao maior período de crescimento (70 dias), foram aplicados $290 \mathrm{mg} \mathrm{dm}^{-3}$ de $\mathrm{N}$, $270 \mathrm{mg} \mathrm{dm}^{-3}$ de $\mathrm{K}$ e $70 \mathrm{mg} \mathrm{dm}^{-3}$ de S. Nos demais tratamentos, com menores períodos de crescimento, as plantas receberam, proporcionalmente, menores doses de $\mathrm{N}, \mathrm{K}$ e S.

Aofinal dos respectivos períodos de crescimento, as plantas foram cortadas aproximadamente a $3 \mathrm{~cm}$ do solo, e subdividiu-se a parte aérea nas frações: fol ha emergente (limbos das fol has não completamente expandidas, ou seja, sem lígula visível), fol ha recémexpandida (limbos das duas folhas recémexpandidas), fol ha adulta (limbos das demais fol has completamente expandidas) e colmo (colmo + bainhas das fol has).

As plantas com a idade de 14 dias de crescimento e sem aplicação de fósforo não foram subdivididas em partes, ao passo que as plantas que receberam as doses de fósfor o foram divididas em apenas fol has recém-expandidas e colmo, em razão de seu pouco crescimento. Pelo mesmo motivo, as plantas com idade de 28 dias e sem aplicação de fósforo também 
não foram subdivididas. Determinou-se o peso da matéria seca $\left(70^{\circ} \mathrm{C}\right)$ de cada uma das frações das plantas. Nos tratamentos em que foram colhidas oi tos plantas, a produção de matéria seca foi corrigida para quatro plantas por unidade experimental.

Todo o material vegetal foi moído em moinho com peneira com abertura de $1 \mathrm{~mm}$ e submetido à mineralização nítrico-perclórica, para dosagem colorimétrica do fósforo (Braga \& Defelipo, 1974). O teor de $\mathrm{P}$ na planta inteira foi obtido por meio de média ponderada entre os teores de fósforo e o peso das frações das plantas.

Antes do transplantio das plântulas, foi feita uma amostragem do sol o em cada unidade experimental, para determinação dos teores de fósforo pel o extrator Mehlich-1 (Defelipo \& Ribeiro, 1981).

Os resultados foram submetidos às análises de variância e de regressão. A cada idade de crescimento das plantas, foram ajustadas equações de regressão, para produção de matéria seca de acordo com as doses de fósforo. A partir dessas equações, estimaram-se as doses necessárias para obtenção de $90 \%$ da produção máxima. Do mesmo modo, estabel eceram-se as regressões para os teores de fósforo na parte aérea de acordo com as doses de fósforo. A recuperação de fósforo pelo extrator Mehlich-1 foi obtida por meio da regressão ajustada entre os teores de fósforo extraídos e as doses de fósforo.

Com base nas doses de fósforo associadas a 90\% da produção máxima enas regressões que relacionam os teores de fósforo no sol o recuperado pel o extrator Mehlich-1 e na parteaérea das plantas, estimaramse os níveis críticos de fósforo no solo e na planta, respectivamente.

\section{Experimento 2 - "rebrotação"}

Neste experimento, realizado simultaneamente com o experimento 1, os tratamentos corresponderam à combinação fatorial entreas duas espécies: as duas idades de rebrotação (28 e 42 dias) de plantas cortadas a $3 \mathrm{~cm}$ do solo, após período inicial de crescimento de 42 dias, e as cinco doses de fósforo aplicadas como foi descrito anteriormente. Os tratamentos foram distribuídos em blocos ao acaso, com três repetições.

As adubações com potássio, nitrogênio e enxofre foram efetuadas a partir do transplantio das plântulas, por meio de aplicações semanais de solução à base de fontes descritas anteriormente, totalizando-se $290 \mathrm{mg} \mathrm{dm}^{-3}$ de N, $270 \mathrm{mg} \mathrm{dm}^{-3}$ de K e $70 \mathrm{mg} \mathrm{dm}^{-3}$ deS, na idade de rebrotação de 28 dias. $\mathrm{Na}$ idade de rebrotação de 42 dias, as plantas receberam maiores doses de $\mathrm{N}$ e K, correspondendo a 310 e $350 \mathrm{mg} \mathrm{dm}^{-3}$, respectivamente.

Os demais procedimentos adotados neste experimento foram iguais aos descritos para o experimento de crescimento inicial.

\section{RESULTADOS E DISCUSSÃO}

\section{Produção de matéria seca da parte aérea das plantas}

Com exceção da $B$. decumbens na idade de 14 dias, a equação quadrática base raiz quadrada foi a que mel hor explicou a variação na produção de matéria seca conforme a aplicação das doses de fósforo (Quadro 1). A partir dessas equações, estimaram-seos $90 \%$ dos rendimentos dematéria seca máximos - o que, em trabal hos de casa de vegetação, corresponde às produções de máxima eficiência econômica (MEE) (Alvarez V., 1996) - e às doses de fósforo associadas a esses rendimentos (Quadro 2).

As estimativas (Quadro 2) evidenciaram que a produção de matéria seca de $B$. decumbens foi, em média, maior tanto no crescimento inicial quanto na rebrotação. Esses resultados deveram-se, essencialmente, à maior produção de $\mathrm{B}$. decumbens verificada nos períodos mais avançados de aval iação em ambos os experimentos. Embora ratifiquem a tendência verificada por Rao et al. (1995), que trabal haram com sete gramíneas forrageiras em dois níveis de fertilidade, esses resultados não eram esperados, uma vez que a espécie P. maximum mostrou maior capacidade de produção de biomassa do quea espécie B. decumbens (Corsi \& Santos, 1995; Leite \& Euclides, 1994). Durante os experimentos, verificou-se que as duas gramíneas apresentaram sintomas visuais de deficiência de nitrogênio e de potássio, apesar da aplicação semanal desses nutrientes, ressaltando que as plantas de $P$. maxi mum expressaram deficiência mais cedo e com maior severidade. Assim, a maior produção de B. decumbens pode ter sido devida à menor exigência e, ou, à maior eficiência nutricional desses nutrientes.

Com relação ao crescimento inicial, verificou-se que as doses de fósforo necessárias para 90\% da produção máxima de P. maximum foram menores, mesmo aos 14 e 28 dias, quando esta espécie teve maior produção (Quadro 2). E mbora aos 28 dias de idade a produção de $\mathrm{B}$. decumbens tenha sido $20 \%$ inferior à deP. maxi mum, a dose de fósforo requerida para MEE foi $38 \%$ maior. Até os 28 dias de crescimento, P. maximum mostrou maior eficiência de aquisição e, ou, de utilização de fósforo. Aos 28 dias de rebrotação, essa espécie também produziu mais quea $B$. decumbens, embora as doses de fósforo associadas à MEE tenham sido praticamenteiguais, o que contribui para confirmar a maior eficiência nutricional de P. maximum quanto ao fósforo em estádio inicial de crescimento.

\section{Fósforo recuperado pelo extrator Mehlich-1}

Obtiveram-se maiores taxas de recuperação do fósforo pel o extrator Mehlich-1 com o aumento das doses aplicadas, sendo o modelo quadrático o que melhor se ajustou aos dados (Figura 1). 
Quadro 1. Equações de regressão da produção de matéria seca $(\mathrm{Y}=\mathrm{g} / \mathrm{vaso})$ de plantas de $\mathrm{B}$. decumbens e P. maximum, considerando as doses de fósforo $\left(X=\mathrm{mg} \mathrm{dm}^{-3}\right)$, e respectivos coeficientes de deter minação $\left(R^{2}\right)$ em diferentes idades de crescimento inicial e de rebrotação

\begin{tabular}{|c|c|c|}
\hline I dade & E quação & $\mathbf{R}^{\mathbf{2}}$ \\
\hline \multicolumn{3}{|l|}{ dia } \\
\hline \multicolumn{3}{|c|}{$\begin{array}{l}\text { Crescimento inicial } \\
\text { Brachiaria decumbens }\end{array}$} \\
\hline 14 & $\hat{\mathrm{Y}}=0,15+0,00578^{* * *} \mathrm{X}-0,0000056 * * * \mathrm{X}^{2}$ & 0,924 \\
\hline 28 & $\hat{\mathrm{Y}}=-0,63+2,78907 * * * \sqrt{X}-0,0619091 * * X$ & 0,964 \\
\hline 42 & $\hat{\mathrm{Y}}=-0,11+7,70041 * * * \sqrt{\mathrm{X}}-0,178493 * * * \mathrm{X}$ & 0,961 \\
\hline 56 & $\hat{\mathrm{Y}}=4,94+13,0588^{* * *} \quad \sqrt{\mathrm{X}}-0,317585^{* * *} \mathrm{X}$ & 0,980 \\
\hline 70 & $\hat{\mathrm{Y}}=13,46+17,0584 * * * \quad \sqrt{ } \mathrm{X}-0,430917 * * * \mathrm{X}$ & 0,970 \\
\hline \multicolumn{3}{|c|}{ Panicum maximum } \\
\hline 14 & $\hat{\mathrm{Y}}=-0,03+0,222947 * * * \sqrt{\mathrm{X}}-0,00423128 * * * \mathrm{X}$ & 0,906 \\
\hline 28 & $\hat{\mathrm{Y}}=1,12+3,91473 * * * \quad \sqrt{\mathrm{X}}-0,101430 * * * \mathrm{X}$ & 0,939 \\
\hline 42 & $\hat{\mathrm{Y}}=4,26+7,53169 * * * \quad \sqrt{ } \mathrm{X}-0,200157^{* * *} \mathrm{X}$ & 0,895 \\
\hline 56 & $\hat{\mathrm{Y}}=8,30+12,1493^{* * *} \quad \sqrt{\mathrm{X}}-0,323643^{* * *} \mathrm{X}$ & 0,952 \\
\hline 70 & $\hat{\mathrm{Y}}=13,77+15,7769 * * * \quad \sqrt{X}-0,419507 * * * X$ & 0,959 \\
\hline \multicolumn{3}{|c|}{ Rebrotação } \\
\hline \multicolumn{3}{|c|}{ Brachiaria decumbens } \\
\hline 28 & $\hat{\mathrm{Y}}=5,79+2,17206 * * * \sqrt{X}-0,0541767 * * * X$ & 0,921 \\
\hline 42 & $\hat{\mathrm{Y}}=11,59+5,89267 * * * \sqrt{ } \mathrm{X}-0,145721 * * * \mathrm{X}$ & 0,960 \\
\hline \multicolumn{3}{|c|}{ Panicum maximum } \\
\hline 28 & $\hat{Y}=6,30+2,5996 * * * \sqrt{ } X-0,0652145 * * * X$ & 0,920 \\
\hline 42 & $\hat{\mathrm{Y}}=12,62+5,1978 * * * \sqrt{ } \mathrm{X}-0,142928 * * * \mathrm{X}$ & 0,936 \\
\hline
\end{tabular}

Quadro 2. Produção de matéria seca da parte aérea de B. decumbens e de P. maximum, correspondente a $90 \%$ da produção máxima, e a respectiva dose de fósforo necessária para obtê-la nas diferentes idades de crescimento inicial e de rebrotação

\begin{tabular}{|c|c|c|}
\hline Idade & Matéria seca & Dose de fósforo \\
\hline dia & $\begin{array}{l}\text { g/vaso } \\
\text { Crescimento inicial } \\
\text { Brachiaria decumbens }\end{array}$ & $\mathrm{mg} \mathrm{dm}^{-3}$ \\
\hline $\begin{array}{l}14 \\
28 \\
42 \\
56 \\
70\end{array}$ & $\begin{array}{r}1,5 \\
27,7 \\
74,6 \\
125,3 \\
164,0\end{array}$ & $\begin{array}{l}344,6 \\
239,4 \\
217,7 \\
194,3 \\
176,6\end{array}$ \\
\hline $\begin{array}{l}14 \\
28 \\
42 \\
56 \\
70\end{array}$ & $\begin{array}{c}\text { Panicum maximum } \\
2,6 \\
35,0 \\
67,6 \\
110,1 \\
145,9\end{array}$ & $\begin{array}{l}326,1 \\
171,8 \\
161,0 \\
159,3 \\
158,4\end{array}$ \\
\hline \multicolumn{3}{|c|}{ Rebrotação } \\
\hline $\begin{array}{l}28 \\
42\end{array}$ & $\begin{array}{c}\text { Brachiaria decumbens } \\
24,8 \\
64,0\end{array}$ & $\begin{array}{l}166,7 \\
175,0\end{array}$ \\
\hline $\begin{array}{l}28 \\
42\end{array}$ & $\begin{array}{c}\text { Panicum maximum } \\
29,0 \\
53,9\end{array}$ & $\begin{array}{l}166,5 \\
137,1\end{array}$ \\
\hline
\end{tabular}

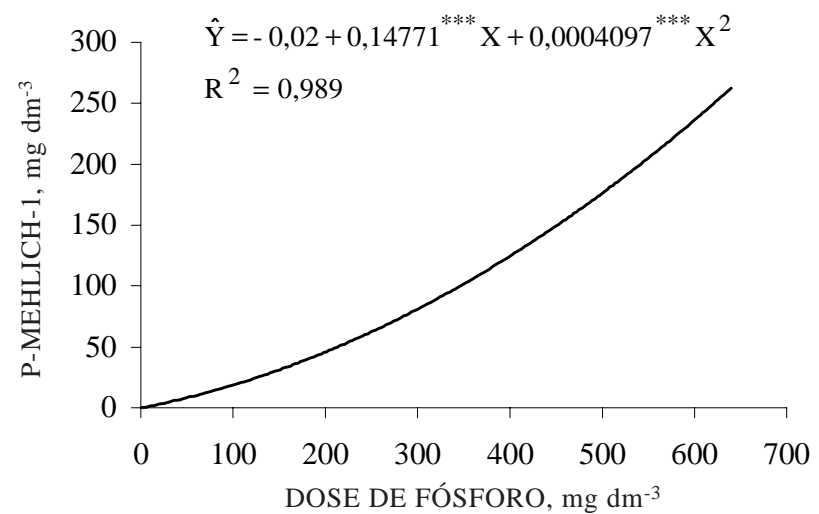

Figura 1. Variação dos teores de fósforo recuperados pelo extrator Mehlich-1, conforme as doses de fósforo aplicadas (*** significativo a $0,1 \%$ ).

\section{Níveis críticos de fósforo no solo}

A variação dos níveis críticos (NíCrí) de fósforo no solo conforme a idade das plantas das duas espécies, durante os períodos de crescimento inicial e de rebrotação, é representada na figura 2 . Tanto para o crescimento inicial quanto para a rebrotação, os valores estimados de NíCrí de fósforo no solo 
foram maiores em B. decumbens (Figura 2), apesar deser a espécieP. maximum mais exigente (Sanchez \& Salinas, 1981). Diferenças de NíCrí entreespécies já eram esperadas, por refletirem as características da espécie quanto à exigência nutricional e às eficiências de aquisição e de utilização de fósforo e outros nutrientes (Neves, 1983).

Considerando quea resposta das plantas às doses de um nutriente depende também da ação e interação de outros fatores, como os demais nutrientes (Alvarez V., 1996), as deficiências de N e de $K$ verificadas em $P$. maximum podem ter contribuído para a menor produção desta gramínea e, conseqüentemente, subestimado a dose de fósforo para atingir a produção de MEE e dos NíCrí no solo.

Os NíCrí defósforo diminuíram exponencial mente com a idade das plantas, ajustando-se a uma regressão potencial (Figura 2), tendência também relatada por Novais et al. (1982) em eucalipto. Na idade de 14 dias na fase de crescimento inicial, estimaram-se valores de NíCrí de fósforo de 95 e $76 \mathrm{mg} \mathrm{dm}^{-3}$ para B. decumbens e P. maximum, respectivamente. Esses valores foram elevados, se comparados àqueles estimados para gramíneas forrageiras, tanto em condições de casa de vegetação quanto de campo (Fonseca et al., 1988; Guss et al., 1990; Corrêa \& Haag, 1993; Fonseca et al., 1997). No entanto, aos 28 dias, os valores de NíCrí foram, em média, 33\% menores para as duas espécies. A partir dessas idades, as quedas nos valores dos NíCrí foram menores, tendendo a estabilizar a partir dos 42 dias de crescimento.

Na fase de rebrotação, mesmo com o corte drástico realizado e com a remoção de praticamente todo o fósforo acumulado na parte aérea das plantas, os NíCrí apresentaram valores próximos aos estimados a partir dos 42 dias de crescimento inicial (Figura 2), evidenciando pequena variação entre as duas idades de rebrotação, o que reforça a tendência de estabilização dos NíCrí com o avanço da idade das plantas.

A diminuição dos valores de NíCrí de fósforo no solo, com o envel hecimento das plantas ou com a sucessão de cortes, tem sido observada por outros autores em eucalipto (Novais et al., 1982), em gramíneas (Barrow, 1975; Fonseca et al., 1988; Guss et al., 1990) e em leguminosas forrageiras (Rivas Y., 1997). Essa tendência tem sido atribuída a três fatores: (a) às alterações na cinética de absorção desse nutriente (J ungk \& Barber,1975; Edwards \& Barber, 1976), principalmente quanto à diminuição da constante cinética de absorção $C_{\text {míni }}$ (b) às associações das raízes com fungos micorrízicos (Urquiaga et al., 1998), e (c) ao aumento do sistema radicular (Schachtman et al., 1998; Barber, 1980), sendo esses dois últimos fatores consi derados os mais importantes, por ser a difusão do fósforo atéàs raízes mais limitante do que a capacidade de absorção destas (Schachtman et al., 1998; Barber, 1980).

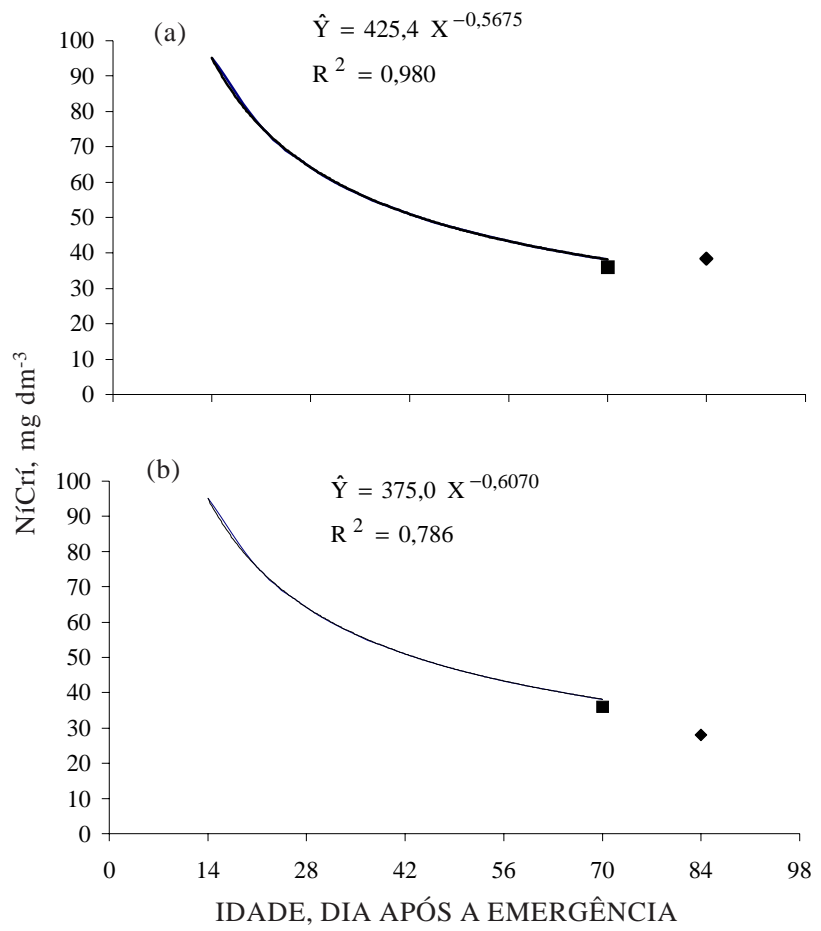

Figura 2. Variação dos níveis críticos de fósforo extraídos por Mehlich-1, de acordo com a idade das plantas deB. decumbens (a) ede P. maximum (b). Os pontos correspondem aos valores de rebrotação de 28 ( $($ ) e 42 ( $\bullet$ ) dias após 42 dias de crescimento inicial.

A taxa específica de absorção de fósforo (TEAF) (g de $\mathrm{P} / \mathrm{g}$ de MS de raízes/dia) do azevém, segundo Barrow (1975), el evou-se, rapidamente, dos 9 aos 15 dias após a emergência das plantas, atingindo o valor máximo e, então, diminuindo lentamente até os 33 dias. Consi derando o comportamento daTEAF, pressupôs-se, inicialmente, que os NíCrí de fósforo fossem elevados para atender à alta demanda metabólica das plantas na fase inicial de desenvolvimento (Marschner, 1995), quando a capacidade de aquisi ção de fósforo era limitada por causa do pequeno sistema radicular. Posteriormente, com o aumento da eficiência dos mecanismos de absorção (aumento da TEAF) e do crescimento radicular, percebeu-se rápida queda nos níveis críticos, sem comprometer o suprimento de fósforo. Os NíCrí diminuíram ainda mais e tenderama uma estabilidade com a idade das plantas, visto que a demanda metabólica caiu eo volume expl orado pelo sistema radicular cresceu, compensando, assim, a queda nos valores das TEAF.

\section{Teores de fósforo na parte aérea das plantas}

A idade da planta e a disponibilidade do fósforo no sol o são os fatores que mais influenciam os teores dessenutriente nas plantas (Bates, 1971; Marschner, 1995). Constatou-se que os teores de fósforo nos 
constituintes da parteaérea das duas espécies, tanto no crescimento inicial quanto na rebrotação, diminuíram com a idade das plantas eaumentaram com as doses de fósforo (dados não apresentados). A diminuição nos teores de fósforo com o envel hecimento das plantas resultou da diluição do teor de fósforo decorrente do acréscimo da proporção de tecido estrutural e de reserva (Marschner, 1995).

Com o desenvolvimento da planta, em geral, os menores teores de fósforo no limbo de folhas de mesma idade fisiológica relacionam-se, segundo Wilson (1976ab), com o aumento da lignificação, da propor ção de esclerênquima e de tecidos vasculares e com a diminuição do tamanho médio do mesofilo em folhas de mesma idade fisiológica, porém em níveis mais el evados de inser ção no perfilho. Graças a redução dos teores de fósforo com a idade em todas as frações da parte aérea, decidiu-se por ajustar equaçõ̃es de regressão entre os teores de fósforo na parte aérea como um todo, conforme as doses de fósforo (Quadro 3).

Quadro 3. Equações de regressão de teores de fósforo na parte aérea $\left(Y=\mathrm{dag} \mathrm{kg}^{-1}\right)$ de plantas de $B$. decumbense de $P$. maximum, considerando as doses de fósforo $\left(X=\mathrm{mg} \mathrm{dm}^{-3}\right)$, e respectivos coeficientes de determinação $\left(R^{2}\right)$ em diferentes idades de crescimento e de rebrotação

\begin{tabular}{lll}
\hline Idade & Equação & $\mathbf{R}^{2}$ \\
\hline
\end{tabular}

dia

Crescimento inicial:

\begin{tabular}{|c|c|c|}
\hline \multicolumn{3}{|c|}{ Brachiaria decumbens } \\
\hline 14 & 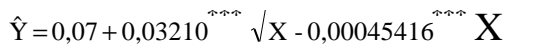 & 0,944 \\
\hline 28 & $\hat{\mathrm{Y}}=0,11+0,00107 \quad \mathrm{X}-0,00000098 \quad \mathrm{X}$ & 0,969 \\
\hline 42 & $\hat{\mathrm{Y}}=0,16-0,00860^{*} \sqrt{\mathrm{X}}+0,00048701^{* * *} \mathrm{X}$ & 0,890 \\
\hline 56 & $\hat{\mathrm{Y}}=0,11-0,00713^{*} \sqrt{\mathrm{X}}+0,00045416^{\text {**** }} \mathrm{X}$ & 0,848 \\
\hline 70 & $\hat{Y}=0,05+0,00025621^{* * * *} \mathrm{X}$ & 0,882 \\
\hline \multicolumn{3}{|c|}{ Panicum maximum } \\
\hline 14 & $\hat{\mathrm{Y}}=-0,16+0,00180^{* * * *} \mathrm{X}-0,00000104^{* * *} \mathrm{X}^{2}$ & 0,954 \\
\hline 28 & $\hat{Y}=0,08+0,00089048 \quad X$ & 0,976 \\
\hline 42 & $\hat{\mathrm{Y}}=0,12-0,01046^{* *} \sqrt{\mathrm{X}}+0,00091262^{* * * *} \mathrm{X}$ & 0,976 \\
\hline 56 & $\hat{\mathrm{Y}}=0,08-0,00940^{* *} \sqrt{\mathrm{X}}+0,00068805^{* * * * \pi} \mathrm{X}$ & 0,961 \\
\hline & $\hat{\mathrm{Y}}=0,05+0,00022272^{* * * *} \mathrm{X}$ & 0,829 \\
\hline
\end{tabular}

Rebrotação:

\begin{tabular}{|c|c|c|}
\hline \multicolumn{3}{|c|}{ Brachiaria decumbens } \\
\hline 28 & $\hat{\mathrm{Y}}=0,20-0,00852^{2-\pi} \sqrt{X}+0,00046273^{\cdots+\pi} \mathrm{X}$ & 0,791 \\
\hline 42 & $\hat{\mathrm{Y}}=0,08-0,00456^{*} \sqrt{ } \mathrm{X}+0,00031035^{* a-1} \mathrm{X}$ & 0,871 \\
\hline \multicolumn{3}{|c|}{ Panicum maximum } \\
\hline 28 & $\hat{\mathrm{Y}}=0,10-0,00590^{\cdots \pi} \sqrt{ } \mathrm{X}+0,00069924^{\pi \cdots \pi} \mathrm{X}$ & 0,981 \\
\hline 42 & $\hat{Y}=0,05+0,00029498^{\cdots \cdots \cdots} x$ & 0,960 \\
\hline
\end{tabular}

Em ambas as espécies, constatou-se que, a partir de 42 dias de crescimento, os teores de fósforo na parte aérea das plantas foram maiores nos tratamentos que não receberam fósforo em relação aos que o receberam nas doses de 64 e $128 \mathrm{mg} \mathrm{dm}^{-3}$. Com a aplicação das maiores doses de fósforo, os teores desse nutriente el evaram-se, caracterizando ofenômeno "Steenbjerg", ou "C shaped curve" (Bates, 1971). Esse efeito foi mais bem visualizado quando serelacionou a produção média de matéria seca, nas idades de 42, 56 e 70 dias, de cada espécie com os respectivos teores médios de fósforo (Figura 3). $\mathrm{Na}$ fase de rebrotação, esse efeito foi menos evidente. $\mathrm{O}$ mesmo fenômeno foi observado por Fonseca et al. (1992) em Hyparrhenia rufa, B. decumbens e Andropogon gayanus. As causas desse fenômeno podem ser atribuídas à falta ou baixa remobilização do fósforo das fol has vel has e dos col mos para tecidos novos, à necrose do meristema apical com a paralisação do crescimento (Marschner, 1995) ou à deficiência severa de fósforo, o que pode diminuir o potencial de crescimento das plantas, mas não o acúmulo de fósforo (Bates, 1971).

\section{Níveis críticos de fósforo na parte aérea das plantas}

Os valores de NíCrí de fósforo na parte aérea diminuíram exponencialmente com a idade das plantas, ajustando-se a uma regressão potencial (Figura 4), à semel hança do verificado em NíCrí de fósforo no solo. I sso indica aumento gradativo da eficiência nutricional das plantas com a idade, envolvendo ganhos na eficiência de aquisição e, ou,

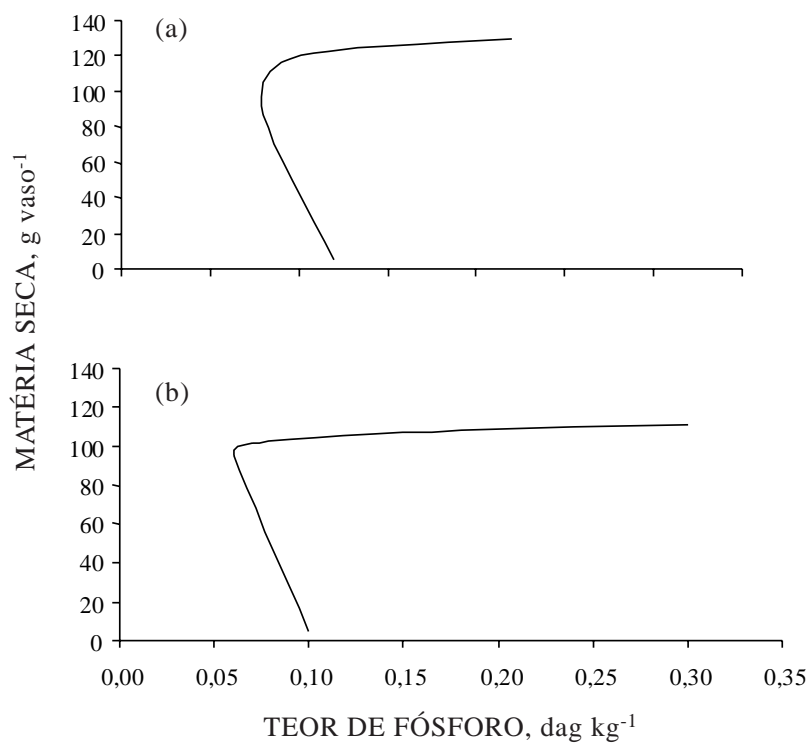

Figura 3. Variação da produção de matéria seca, considerando os teores de fósforo na parte aérea de $B$. decumbens (a) e de P. maxi mum (b). Média dos valores aos 42, 56 e 70 dias. 
transl ocação e, ou, utilização e, conseqüentemente, menor requerimento (interno e externo) desse nutriente.

E mbora as duas espécies tenham apresentado a mesma tendência de variação, a espécie $B$. decumbens exi biu maiores Nícrí em todas as idades (Figura 4), exceto aos 14 dias de crescimento. Os NíCrí de fósforo maiores em $B$. decumbens do que em cultivares da espécieP. maximum também foram encontrados em outros estudos (Martinez \& Haag, 1980; Hoffmann et al., 1995).

$\mathrm{Na}$ idade de 14 dias de crescimento inicial, estimaram-seNíCrí de fósforo de 0,57 e0,60 dag kg-1, para B. decumbens eP. maximum, respectivamente, valores esses considerados el evados e semel hantes aos estimados para espécies olerícolas muito exigentes de fósforo (Novais et al., 1993). No entanto, nas idades seguintes, com a diminuição dos NíCrí, os valores estimados aproximaram-se dos NíCrí estabel ecidos para essas espécies forrageiras em outros trabal hos (Fonseca et al., 1992; Guss et al., 1990; Hoffmann et al., 1995).

Os NíCrí de fósforo na parte aérea das plantas também diminuíram com a idade de rebrotação (Figura 4) eforam similares àquel es estimados para as idades mais avançadas do crescimento inicial. Considerando que houve período de crescimento inicial de 42 dias, as plantas col hidas aos 28 e 42 dias de rebrotação tinham idade de 70 e 84 dias após a emergência, respectivamente. Apesar disso, os níveis críticos estimados para a rebrotação de 28 dias foram mais el evados do que os para as plantas de mesma idade, sem sofrer corte. Como o corte realizado eliminou toda a parte aérea das plantas, a maior proporção de fol has novas e o menor crescimento do caule no período de 28 dias de rebrotação explicam os maiores valores de NíCrí. Aos 42 dias de rebrotação, os NíCrí aproximaram-se dos valores das duas maiores idades no crescimento inicial.

Os menores valores de NíCrí na fase de rebrotação, em relação aos valores estimados para as menores idades do crescimento inicial, ratificaram o aumento da eficiência nutricional do fósforo com a idade das plantas, daí os menores requerimentos de fósforo depois do estabelecimento dessas forrageiras. Dessa forma, para avaliação criteriosa do estado nutricional do fósforo na planta, devem-se levar em consideração a idade das plantas após a implantação e em cada cicl o de crescimento, as características do solo e os demais fatores citados por Bates (1971).

\section{CONCLUSÕES}

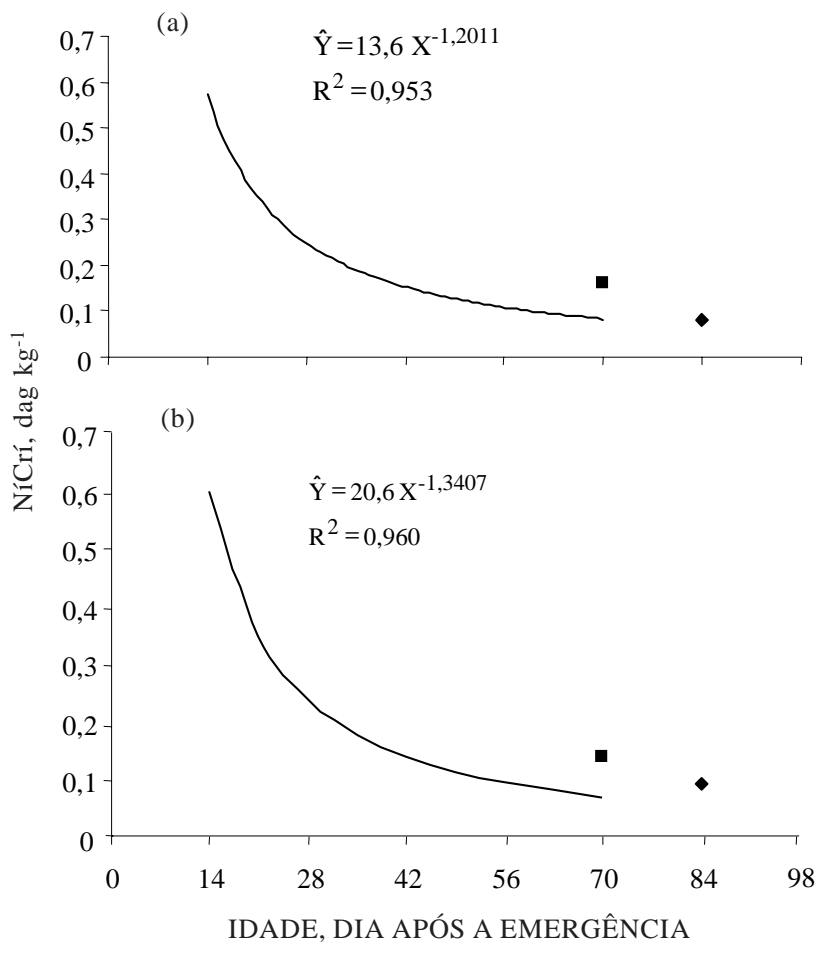

Figura 4. Variação dos níveis críticos de fósforo na parte aérea, consi derando a idade das plantas de B. decumbens (a) e de P. maximum (b). Os pontos correspondem aos valores de rebrotação de 28 (匹) e 42 (४) dias após 42 dias de crescimento inicial.
1. Os NíCrí de fósforo no solo e na parte aérea das plantas das duas espécies de gramíneas forrageiras decresceram exponencialmente de acordo com a idade de crescimento das plantas.

2. Os NíCrí de fósforo no solo, aos 28 e 42 dias de rebrotação, mantiveram-secom val ores próximos aos determinados para a idade de 70 dias no crescimento inicial.

3. Os NíCrí defósforo na parteaérea das plantas, na fase de rebrotação, foram maiores aos 28 dias, mas menores do que os NíCrí estimados nas idades de 14 e 28 dias de crescimento inicial. Aos 42 dias de rebrotação, os NíCrí tiveram valores si milares aos estabelecidos nas idades de 56 e 70 dias no crescimentoinicial.

4. A queda exponencial dos NíCrí de fósforo no solo e na planta evidenciou a redução dos requerimentos desse nutriente conforme a idade de crescimento das plantas. Dessa forma, recomendase o manejo da adubação das duas gramíneas forrageiras: adubação de plantio ou "arranque" e outra de manutenção da produtividade.

\section{LITE RATURA CITADA}

ALVAREZV., V.H. Correlação e cal ibração de métodos de análise desolos. In:ALVAREZV., V.H.;FONTES, L.E.F. \& FONTES, M.P.F., eds. O solo nos grandes domínios morfoclimáticos do Brasil e o desenvolvimento sustentado. Viçosa, Sociedade Brasileira de Ciência do Solo, 1996. p.615-645. 
ALVAREZ V., V.H. \& FONSECA, D.M. Definição de doses de fósforo para determinação da capacidade máxima de adsorsão de fosfatos e para ensaios em casa de vegetação. R. Bras. Ci. Solo., 14:49-55, 1990

ALVAREZV., V.H.; NOVAIS, R.F.; DIAS, L.E. \& OLIVEIRA, J .A. Determinação e uso do fósforo remanescente. B. Inf. SBCS, 25:27-32, 2000.

BARBER, S.A. Soil-plant interactions in the phosphorus nutrition of plants. In: KHASAWNEH, F.E.; SAMPLE, E.C. $\&$ KAMPRATH, E.J., eds. The role of phosphorus in agriculture. Madison, American Society of Agronomy, 1980. p.591-616.

BARROW, N.J . The response to phosphate of two annual pasture species. II The specific rate of uptake of phosphate, its distribution and use for growth. Aust. J. Agric. Res., 26:145-156, 1975.

BATES, T.E. Factors affecting critical nutrient concentrations in plants and their evaluation: a review. Soil Sci., 112:116$130,1971$.

BRAGA， J.M. \& DEFELIPO, B.V. Determinação espectofotométrica do fósforo em extratos de sol os e plantas. R. Ceres, 21:124-136, 1974.

CORRÊA, L.A. \& HAAG, H.P. Níveis críticos de fósforo para o estabelecimento de gramíneas forrageiras em Latossolo Vermelho-Amarelo, álico: I: Ensaio em casa de vegetação. Sci. Agric., 50:99-108, 1993.

CORSI, M., SANTOS, P.M. Potencial de produção do Panicum maximum. In: SIMPÓSIO SOBRE MANEJO DA PASTAGEM, 12., Piracicaba, 1995. Anais. Piracicaba, Fundação de Estudos Agrários Luiz de Queiroz, 1995. p.275-304.

DEFELIPO, B.V. \& RIBEIRO, A.C. Análise química do solo. Viçosa, Universidade Federal de Viçosa, 1981. 15p.

EDWARDS, J.H. \& BARBER, S.A. Phosphorus uptake rate of soybeans roots as influenced by plant age, root trimmed, and solution P concentration. Agron. J ., 68:973-975, 1976.

FONSECA, D.M.; ALVAREZ V., V.H.; NEVES, J .C.L.; GOMIDE, J.A.; NOVAIS, R.F. \& BARROS, N.F. Níveis críticos de fósforo em amostras de solo para o estabelecimento de Andropogon gayanus, Brachiaria decumbens e Hyparrhenia rufa. R. Bras. Ci. Solo, 12:49-58, 1988.

FONSECA, D.M.; GOMIDE, J .A.; ALVAREZ V., V.H.; NEVES, J.C.L.; NOVAIS, R.F. \& BARROS, N.F. Absorção, utilização e níveis críticos de fósforo em Andropogon gayanus, Brachiaria decumbens e Hyparrhenia rufa. R. Soc. Bras. Zoot., 21:730-743, 1992.

FONSECA, D.M.; GOMIDE, J .A.; ALVAREZV., V.H. \& NOVAIS, R.F. Fatores que influenciam os níveis críticos de fósforo para o estabelecimento de gramíneas forrageiras: I. Casa de vegetação. R. Bras. Ci. Solo, 21:27-34, 1997.

GNIAZDOWSKA, A.; KRAWCZAK, A.; MIKULSKA, M. \& RYCHTER, A.M. Low phosphorus nutrition alters beans plants' ability to assimilateand translocatenitrate. J. Plant Nut., 22:551-563, 1999.

GUSS, A.; GOMIDE, J.A. \& NOVAIS, R.F. Exigência de fósforo para o estabelecimento de quatro espécies de Brachiaria em solos com características físico-químicas distintas. R. Soc. Bras. Zoot., 19:278-289, 1990.
HOFFMANN, C.R.; FAQUIN, V.; GUEDES, G.A.A. \& EVANGELISTA, A.R. O nitrogênio e o fósforo no crescimento da braquiária e do colonião em amostras de um Latossolo da região noroeste do Paraná. R. Bras. Ci. Solo, 19:79-86, 1995.

HOLFORD, I.C.R. Soil phosphorus: its measurement, and its uptake by plants. Aust. J. Soil Res., 35:227-239, 1997.

JUNGK, A. \& BARBER, S.A. Plant age and the phosphorus uptake characteristics of trimmed and untrimmed corn root systems. Plant Soil, 42:227-239, 1975.

LEITE, G.G. \& EUCLIDES, V.P.B. Utilização de pastagens de Brachiaria spp. In: SIMPÓSIO SOBRE MANEJ O DA PASTAGEM, 11., Piracicaba, 1994. Anais. Piracicaba, Fundação deE studos Agrários Luiz de Queiroz, 1994. p.267297.

MACEDO, M.C.M. Pastagens no ecossistema cerrado: pesquisa para o desenvolvimento sustentável. In: SIMPÓSIO SOBRE PASTAGENS NOS ECOSSISTEMAS BRASILEIROS: PESQUISAS PARA O DESENVOLVIMENTO SUSTENTÁVEL, Brasília, 1995. Anais. Brasília, Sociedade Brasileira de Zootecnia, 1995. p.28-62.

MARSCHNER, H. Mineral nutrition of higher plants. 2.ed. New York, Academic Press, 1995. 874p.

MARTINEZ, H.E.P. \& HAAG, H.P. Níveis críticos de fósforo em Brachiaria decumbens (Stapf) Prain, Brachiaria humidicola (Rendle) Schweickerdt, Digitaria decumbens Stent, Hyparrhenia rufa (Ness) Stapf, Melinis minutiflora Pal de Beauv, Panicum maximum J acq. e Pennisetum purpureum Schum. An. ESALQ, 37: 913-977, 1980.

NEVES, J.C.L. Aspectos nutricionais em mudas de Eucalyptus spp - Tolerância ao alumínio e níveis críticos de fósforo no solo. Viçosa, Universidade Federal de Viçosa, 1983. 87p. (Tese de Mestrado)

NOVAIS, R.F.; BARROS, N.F.; NEVES, J.C.L. \& COUTO, C. Níveis críticos de fósforo no solo para o eucalipto. R. Arv, 6:29-37, 1982.

NOVAIS, R.F.; NEVES, J .C.L.; BARROS, N.F.; CASALI, V.W.D. $\&$ FABRES, A.S. The influence of soil phosphate capacity factor on soil and plant phosphorus critical levels of different vegetables. In: FRAGOSO, M.A.C. \& VAN BEUSICHEM, M.I., eds. Optimization in plant nutrition. Dordrecht, Kluwer Academic Press, 1993. p.73-76.

RAO, I.M.; AYARZA, M.A. \& GARCIA, R. Adaptive attributes of tropical forage species to acid soils I. Differences in plant growth, nutrient acquisition and nutrient utilization among $\mathrm{C}_{4}$ grasses and $\mathrm{C}_{3}$ legumes. J. Plant Nutr., 18:2135-2155, 1995.

RIVAS Y., F.F. Nutrição fosfatada e fotossíntese no sistema simbiótico Medicago sativa - Rhizobium meliloti em solos com diferentes disponibilidades de fósforo. Viçosa, Universidade Federal de Viçosa, 1997. 124p. (Tese de Doutorado)

SANCHEZ, P.A. \& SALINAS, J.G. Low-input technology for managing oxisols in Tropical America. Adv. Agron., 34:279406, 1981.

SCHACHTMAN, D.P.; REID, R.J . \& AYLING, S.M. Phosphorus uptake by plants: from soil to cell. Plant Physiol., 116:447453, 1998 
URQUIAGA, S.; OLIVEIRA, O.; OLIVEIRA, I.; FERREIRA, E.; ALVES, B.; CADISCH, G. \& BODDEY, R. Soil nutrient availability in relation to the productivity of pastures in the Brazilian cerrado. In: WORLD CONGRESS OF SOIL SCIENCE, 16., Montpellier, 1998. Proceedings. Montpellier, International Soil Science Society, 1998. (Scientific registration, 2291; Symposium, 14; CD-ROM)
ZIMMER, A.H. \& CORREA, E.S. A pecuária nacional, uma pecuária de pasto? In: ENCONTRO SOBRE RECUPERAÇÃO DE PASTAGENS, Nova Odessa, 1993. Anais. Nova Odessa, Instituto de Zootecnia, 1993. p.1-26.

WILSON, J.R. Variation of leaf characteristics with level of insertion on a grass tiller. II Anatomy. Aust. J. Agric. Res., 27:355-364, 1976b. 